\title{
Cerebral cortex: a target and source of insulin?
}

\author{
Éva A. Csajbók ${ }^{1}$ - Gábor Tamás ${ }^{2}$
}

Received: 13 April 2016/Accepted: 29 April 2016/Published online: 20 May 2016

(C) Springer-Verlag Berlin Heidelberg 2016

\begin{abstract}
Recent results suggest that insulin is synthesised by a subpopulation of neurons in the cerebral cortex and neural progenitor cells of the hippocampus. Supplementing the slow supply of insulin to the brain by pancreatic beta cells, the insulin locally released by neurons provides a rapid means of regulating local microcircuits, effectively modulating synaptic transmission and on-demand energy homeostasis of neural networks. Modulation of insulin production by brain neurons via glucagon-like peptide 1 (GLP-1) agonists might be useful in counteracting diabetes, obesity and neurodegenerative diseases. Replacement of lost pancreatic beta cells by autologous transplantation of insulin-producing neural progenitor cells could be a viable therapy for diabetes.
\end{abstract}

Keywords Animal · Basic science $\cdot$ Human - Insulin action Insulin synthesis $\cdot$ Rat $\cdot$ Review

\author{
Abbreviations \\ GLP-1 Glucagon-like peptide 1 \\ LTD Long-term depression \\ LTP Long-term potential \\ MAPK Mitogen-activated protein kinase
}

Gábor Tamás

gtamas@bio.u-szeged.hu

1 Endocrine Unit, 1st Department of Internal Medicine, University of Szeged, Szeged, Hungary

2 MTA-SZTE Research Group for Cortical Microcircuits, Department of Physiology, Anatomy and Neuroscience, University of Szeged, Közép Fasor 52, Szeged 6726, Hungary

\section{Introduction}

Discovered almost a century ago [1], insulin is widely known for its essential role in reducing blood glucose levels through the stimulation of glucose uptake by muscle, liver and adipose tissues. Pancreatic beta cells secrete insulin, a process that is crucial for determining blood glucose levels. Beta cells detect extracellular glucose concentrations through the glucose transporter GLUT2. Importantly, unlike the glucose transporters in insulinsensitive tissues (predominantly GLUT4), GLUT2 is not regulated by insulin, thus the drop in blood glucose level due to the action of GLUT4 triggers a negative feedback mechanism suppressing insulin secretion through GLUT2 positioned on beta cells. Type 1 diabetes mellitus is a consequence of the partial or complete loss of beta cells, which leads to impaired insulin secretion and uncontrolled blood glucose levels (hypoinsulinaemic hyperglycaemia). In type 2 diabetes, insulin levels are indirectly disturbed. Insufficient insulin action on receptors in insulinsensitive tissues allows blood glucose levels to rise, leading to enhanced insulin release by beta cells. This insulin release is initially capable of restoring normal blood glucose concentrations (hyperinsulinaemic euglycaemia). When beta cells reach their maximal insulin-producing capacity and are no longer able to maintain normal blood glucose, the state of hyperinsulinaemic hyperglycaemia known as type 2 diabetes arises.

The function of insulin, however, is not restricted to peripheral organs. In the brain, the insulin-independent GLUT1 and GLUT3 are predominantly responsible for glucose uptake in glial cells and neurons, respectively [2]. Accordingly, brain metabolism has been considered insulin independent for decades, but the discovery of insulin receptors in the brain [3] now indicates that the cerebral functions of insulin are more complex. Insulin receptors expressed in the brain are similar to those found in the periphery [4], with the highest expression detected in the olfactory bulb, cerebral cortex, hippocampus, 
hypothalamus and cerebellum [3]. Insulin receptor levels are higher in neurons than in glial cells [5]. Importantly, there are similarities in the periphery and the brain in the major signal transduction pathways linked to the insulin receptor. The phosphoinositide-3 kinase (PI3)/Akt and Ras/mitogenactivated protein kinase (MAPK) routes crucial to peripheral glucose, lipid and protein metabolism [6] are also essential in the mechanism of insulin action in the brain $[4,7]$.

This short paper reviews the classic ideas emphasising the role of pancreatic insulin in the central nervous system [8], shares the conclusion of excellent recent reviews [9-11] by arguing that local insulin synthesis in the brain is significant and finally presents potential therapies targeted to or based on insulin synthesised by neurons or their progenitors.

\section{External insulin reaches the brain}

As outlined above, a normal supply of insulin in the brain appears to be crucial for neural function, including metabolism, and, consequently, dynamic or persistent alterations in insulin-dependent mechanisms could contribute to pathological processes. The sources of insulin found in the brain are not completely clear. It is generally accepted that insulin synthesised by pancreatic beta cells is delivered to the brain $[8$, 12-16], but an accurate picture of this process is missing (Fig. 1) [10]. Pancreatic insulin circulating in the plasma has two ways into the interstitial fluid immediately surrounding neurons and glial cells of the brain.

The first pathway delivers relatively small amounts of plasma insulin through the choroid plexus to the cerebrospinal fluid. Plasma concentrations of insulin are an order of magnitude higher compared with those measured in the cerebrospinal fluid $[17,18]$. Interestingly, this difference is increased in obesity [19], despite the higher plasma insulin concentrations in obese individuals. This process is saturable [16,20], but it is not clear whether saturation is caused by the potential involvement of insulin receptors of the choroid plexus or by the suspected contribution of megalin, a transporter known to mediate leptin transport across the choroid plexus and to be involved in insulin transport [21] in the epithelial cells of renal tubules [22].

The second pathway takes insulin from plasma into the endothelial cells of the brain microvasculature. Based on experiments showing that aortic endothelial cells outside the brain are able to concentrate insulin [23], the second pathway is hypothesised to transport the bulk of peripheral insulin to the brain. Mechanisms of transendothelial insulin transport in the brain have not been directly studied to date, but one can speculate that a vesicular trafficking process, beginning with insulin binding to its receptor followed by the involvement of caveolae and promoted by NO signalling [23-26], could be involved according to experiments with peripheral endothelia. The two pathways join at the Virchow-Robin space surrounded by endothelial cells, astrocytic endfeet and pericytes. Peripheral insulin then passes the line of astrocytic endfeet, an effective filter and limiter of movement speed for larger molecules [27], before reaching the interstitial space around neurons and glia. Absolute insulin concentrations are difficult to measure reliably in the interstitial space of the brain, but the relative changes detected in response to food intake were independent of plasma insulin concentration $[28,29]$, raising the possibility that pancreas-independent local insulin synthesis occurs in the brain [13].

The speed of pancreatic insulin transport into the cerebrospinal fluid and then to the interstitial space of the brain is orders of magnitude slower than the operating speed of neural networks estimated according to the lowest frequency of brain oscillations. Insulin levels in the cerebrospinal fluid remain below fasting levels in response to several hours of peripheral hyperinsulinaemic euglycaemic clamping $[18,20]$ and, moreover, fasting insulin levels in the cerebrospinal fluid $(\sim 7 \mathrm{pmol} / \mathrm{l})$ [30] are insufficient for signal transduction through insulin receptors. Even if insulin concentrations in the cerebrospinal fluid are elevated to effective levels, it is estimated that the slow circulation of cerebrospinal fluid limits insulin delivery to the interstitial space of the brain at a rate of $\sim 1 / 600$ of that to skeletal muscle and at $<1 / 30,000$ of that to the liver [10]. Alternatively, insulin might move directly from the plasma through the blood-brain barrier to the Virchow-Robin space and the interstitial fluid. Studies examining the involvement of
Fig. 1 Potential central actions of insulin. The box represents the blood-brain barrier

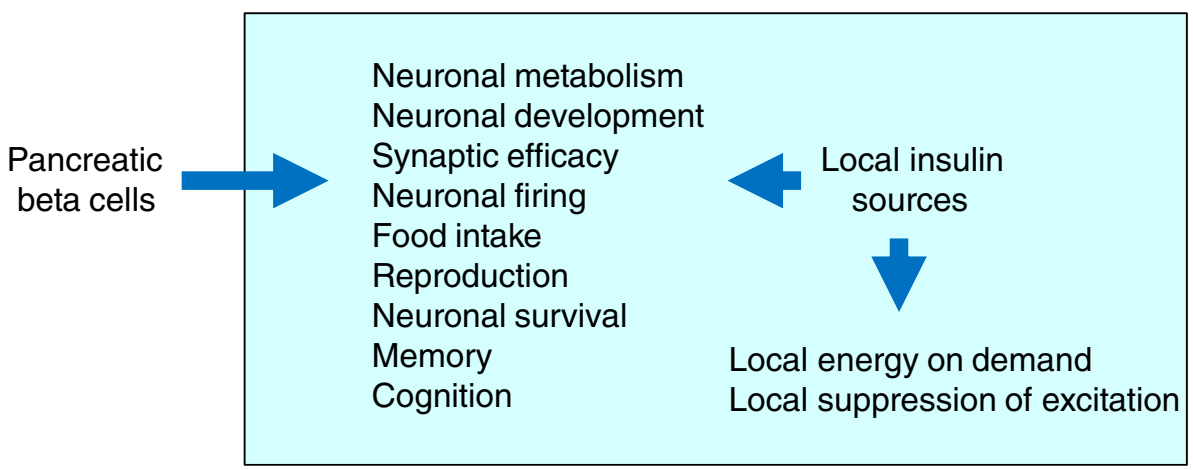


this route measured the tissue content of radiolabelled insulin in brain regions [31], which did not allow the insulin concentration in the interstitial fluid to be determined. To date, estimations of the speed by which insulin moves across the bloodbrain barrier are limited by brain microvessels binding insulin with high affinity without significant insulin degradation [32]. Nevertheless, insulin finds its way from the plasma to the immediate vicinity of neurons, but equilibration with the interstitial space in the brain is achieved at timescales consistent with long-term homeostatic regulation outside the frequency range $(\sim 0.1-200 \mathrm{~Hz})$ of changes in membrane potential in neural networks.

The limited speed by which external insulin is distributed is also a factor to consider when delivering insulin to the brain intranasally [30]. This process has gained particular relevance following encouraging reports [33] and clinical trials [34] that provide evidence for cognitive improvements with daily intranasal insulin administration in patients with mild cognitive impairment or mild to moderate Alzheimer's disease. Levels of insulin are reduced in Alzheimer's disease [35, 36], and intranasally applied insulin raises concentrations in the cerebrospinal fluid within $10 \mathrm{~min}$ of application, with maximal levels achieved after $30 \mathrm{~min}$, while plasma insulin and glucose levels remain unaffected [30]. How intranasal insulin reaches the brain remains mechanistically unclear [37], but the process can be stimulated by inhibiting protein kinase $\mathrm{C}$ [38]. A different strategy for increasing insulin concentrations in key areas affected by Alzheimer's disease, such as the hippocampus and neocortex, would be to boost insulin release from neurons or neuronal progenitors expressing insulin locally.

\section{Local insulin synthesis in the brain}

Whether insulin is produced locally in the central nervous system is not a trivial question to answer. Initial studies on the subject suggested that immunoreactive insulin is present in the rat brain in concentrations 10 to 100 times higher than in the plasma [39], but this was challenged by subsequent findings [40], leading to the conclusion that 'little or no insulin is produced in [the] brain' [41]. The heart of the problem is that experiments must be able to differentiate between insulin of pancreatic origin and insulin synthesised locally. Anti-insulin antibodies recognise the same epitopes on pancreas- and brain-derived insulin, thus methods such as anti-insulin immunocytochemistry or radioimmunoassay capable of detecting insulin in small amounts are not adequate. Increasing the resolution to allow cellular or subcellular localisation of antiinsulin immunoreaction signals is of little help as receptorbound and internalised insulin pools are degraded or recycled to the plasma membrane at intracellular locations that potentially overlap with those of locally synthesised peptide [42].
Immunoreactions detecting peptides involved in the different steps of insulin synthesis might overcome these limitations. Indeed, C-peptide, an integral part of proinsulin, was localised to the same neurons as insulin [43-45], and proinsulin-like immunoreactivity was documented in samples derived from the central nervous system [46], arguing for local synthesis in the brain.

Another strategy for detecting insulin production in the brain is to search for the mRNA of insulin-coding genes: Ins 1 and Ins 2 in mice; Ins 2 in rat; and INS in humans. A pioneering RT-PCR study detected widespread Ins 2 expression in the rat brain throughout development [47] and the same laboratory confirmed it in rabbit, showing Ins 2 expression in neurons of the hippocampus and olfactory bulb [48]. More recently, hippocampal granule cells from adult rats and neuronal progenitor cells derived from the hippocampus or olfactory bulb were also found to express insulin mRNAs [45]. Furthermore, expression of Ins 2, but not Ins 1, was found in cortical and subcortical areas of the mouse brain [49, 50] and INS mRNA expression characterised human samples of the hippocampus, amygdala and temporal lobe in addition to the olfactory bulb, cerebellar and pontine regions [50]. Recently developed methods to precisely quantify mRNA copy numbers in single neurons [51] have provided an effective tool for determining Ins 2 levels in several rat neuron types and astrocytes in the rat cerebral cortex [52]. Interestingly, a subset of inhibitory GABAergic neurons, the so-called neurogliaform interneurons, expressed Ins 2 mRNA at the highest copy numbers tested, excitatory pyramidal neurons contained Ins 2 mRNA at low copy numbers, and other GABAergic neurons and astroglial cells did not express Ins 2 mRNA above the threshold of detection [52]. Importantly, the authors found that mRNA copy number was selectively increased in response to increasing extracellular glucose concentrations in the cell types expressing Ins 2 [52], suggesting that neuronal production of insulin could be associated with local metabolic supply and functional demand, especially in neocortical and hippocampal areas of the cerebral cortex.

The first experiments showing local release of insulin in the cerebral cortex followed classic ideas of mimicking the effect of externally added compounds with endogenously released substances. In this case, Molnár et al [52] first determined that external insulin is effective in suppressing spontaneous excitatory potentials arriving at neurons of the neocortex, then, using local delivery of glucose or glibenclamide to neurogliaform interneurons (known to express Ins 2 mRNA, see above), forced the release of an endogenous substance that also suppressed spontaneous excitatory potentials. Finally, they blocked this effect with the specific insulin receptor antagonist S961, revealing the identity of the endogenous substance as insulin. Thus, insulin can be released from a subpopulation of inhibitory neurons of the cerebral cortex and has an excitationsuppressing effect in local neural microcircuits. 


\section{Insulin action in the brain}

Given the parallel expression of insulin-activated PI3/Akt and Ras/MAPK pathways in the periphery and the brain, a logical question is whether insulin-mediated effects on neurons involve metabolic regulation. Insulin receptors and the insulinsensitive glucose transporter GLUT4 have been shown to colocalise on neurons [53], and cellular mechanisms supporting neuronal metabolic functions of insulin involve translocation of GLUT4 to the cell surface [54], so providing an alternative to insulin-independent glucose uptake through GLUT3. The insulin dependence of brain metabolism at the neural network level has also been revisited by a number of human in vivo studies [55-57], which suggest that insulin can effectively stimulate glucose uptake in the medial temporal lobe, especially during periods of intensive neuronal activity [58]. Moreover, a rapid increase in local glycolysis following insulin administration was found in the hippocampus and was suppressed in type 2 diabetes [59]. However, glucose levels drop significantly in the extracellular space during intense cognitive operations and might not be rate-limiting for the metabolic supply of microcircuits because of astrocytic metabolic routes [11]. A consensus on neuronal glucose metabolism in relation to insulin is of particular interest for two reasons. First, neuronal ensembles in the hippocampus and the neocortex are engaged in increased high-frequency epochs of firing during memory formation or cognitive tasks and the extra metabolic demand created by intensive action potential generation might be met by alternative routes of supply. An unorthodox pathway of glucose supply during cognitive surges in energy demand was suggested by Emmanuel et al [58], who proposed that non-insulin-dependent GLUT1 and GLUT3 transport is sufficient for resting brain activity, while sustained cognitive activity induces the addition of insulinsignalled GLUT4 transport. Second, unlike in other organs, glucose is central for the energy metabolism of the brain and temporary or sustained changes in glucose supply could be crucial in differentiating the normal and pathological functions of neural circuits. Cognitive deficits are associated with insulin resistance and diabetes $[60,61]$ and impaired insulindependent mechanisms for glucose uptake during tasks requiring extra supply might lead to deficient energy metabolism [58]. Along the same vein, 'type 3 diabetes' was suggested as an alternative term for Alzheimer's disease [35], based on observations showing reduced insulin expression and signalling mechanisms in the sporadic form of the disease [62].

In addition to the involvement in neuronal metabolism, the classic PI3/Akt and Ras/MAPK insulin-activated pathways are also important in neuron-specific cellular functions, such as the development of neuronal dendritic arbors [63] and the maintenance of excitatory synapses [64, 65]. These functions are crucial in regulating cellular processes of learning and memory and lead to long-term potentiation (LTP) or long-term depression (LTD) of synaptic efficacy through the removal of $\alpha$-amino-3-hydroxy5-methyl-4-isoxazolepropionic acid (AMPA) receptors from postsynaptic specialisations for LTD $[64,65]$ and through shifting the stimulation frequency-response function for LTP [66]. Corroborating these results, a deficit of LTP is observed in models of type 1 diabetes, but results from experiments using type 2 diabetes model systems are not unequivocal [54]. Insulin is instrumental in modulating inhibitory mechanisms by moving additional $\gamma$-aminobutyric acid (GABA) receptors to inhibitory synapses [67] and extrasynaptic regions of the plasma membrane [68], providing mechanisms synergistic with the LTD induction detailed above for shifting the balance away from excitation in neural networks.

Neurogliaform interneurons, capable of releasing insulin in cortical microcircuits [52], are also GABAergic [69] and use GABA for volume transmission of widespread inhibition [70], thus these cells are ideally suited to synchronise the local actions of insulin and GABA. It is not yet known which combination of neural afferents elicit insulin release from neurogliaform cells. However, it is reasonable to assume that strong excitatory inputs might contribute to the intracellular $\mathrm{Ca}^{2+}$ accumulation required for peptide release. One can speculate that insulin release could be synchronised to elevated overall activity in networks around neurogliaform neurons. This way transient local energy demand could be met by insulin release-driven additional glucose transport through insulindependent GLUT4, as suggested for epochs of intense hippocampal or cortical activity during cognitive processing [58]. At the same time, the overall excitation-suppressing activity of insulin released from neurogliaform cells is likely to be coupled with the synchronous release and inhibitory action of GABA from neurogliaform cells, which might curtail energy demand.

At the neuronal network level, insulin is an effective neuromodulatory peptide with an array of effects including control of food intake and body weight, regulation of the reproductive or hypothalamic-pituitary-gonadal axis, influencing neuronal survival and modulation of memory and cognitive processes [8, 12-14]. Apart from the classic cellular signalling pathways, insulin also acts through alternative mechanisms in neurons. Insulin directly opens neuronal ATP-gated potassium $\left(\mathrm{K}_{\mathrm{ATP}}\right)$ channels, resulting in suppressed firing [71], and this signalling route was suggested as a mechanism for modulating hippocampal memory performance [72]. Moreover, the action of insulin on neurons might interact with glucocorticoid signalling through opposite modulation of hippocampal GLUT4 [73] and by additional mechanisms expertly reviewed previously $[11,54]$.

\section{Neuron-derived insulin-based therapy}

The effect of glibenclamide in triggering neuronal release of insulin [52] also suggests that the delivery of substances known to enhance insulin release from pancreatic beta cells to the brain 
might have therapeutic implications. An as-yet untested strategy for increasing insulin concentrations in key areas affected by Alzheimer's disease, such as the hippocampus and neocortex, would be to boost insulin release from neurons or neuronal progenitors expressing insulin locally. Apart from sulfonylureas, incretins might represent a promising group of molecules to be tested for several reasons. Glucagon-like peptide 1 (GLP1) receptors are expressed in neurons of the hippocampus and the neocortex [74], although the expression of GLP-1 receptors has not been documented on insulin-expressing neurons or neural progenitor cells. Interestingly, however, GLP-1 agonists have effects on tonic inhibitory GABAergic currents similar to those reported for insulin, arguing for a hypothetical contribution of GLP-1 receptor-mediated insulin release [68, 75]. GLP1 is produced in the brainstem [76], suggesting that centrally synthesised GLP-1 could be effective within the brain via the mechanisms outlined above. However, GLP-1 produced by Lcells of the intestine crosses the blood-brain barrier [76] and thus incretins arriving from the periphery can possibly enhance insulin release from neurons in the brain. Importantly, these peripheral incretins include GLP-1 analogues prescribed in type 2 diabetes mellitus. We suggest that the weight loss caused by GLP-1 receptor analogue-based therapy (attributed primarily to the inhibition of gastric emptying [77]) might have an additional synergistic component through GLP-1 receptor-mediated insulin release from neurons of the brain. Human imaging studies suggest that the prefrontal cortex is crucial in the inhibitory control of food intake [78-80] and, hypothetically, expression of GLP-1 receptors on insulin-releasing neurogliaform neurons of the prefrontal cortex could provide mechanistic support for this process. Moreover, GLP-1 receptor agonists promise therapeutic effectiveness against neurodegeneration in models of Alzheimer's, Huntington's and Parkinson's diseases [81, 82] and a scenario of GLP-1 receptor-mediated insulin synthesis in the brain could be extended to therapy for these diseases.

The evidence for insulin synthesis in the brain raises the question of whether brain-derived insulin could be used to replace peripheral insulin in type 1 diabetes. Insulin synthesised in the brain is unlikely to cross the blood-brain barrier in the brain-toblood direction in the quantity required for euglycaemic control of plasma glucose concentrations [8]; intranasal insulin delivery fails to increase plasma insulin levels significantly [30]. An alternative approach might use autologous grafts of insulinexpressing neurons or neural progenitor cells as a potential replacement for lost pancreatic beta cells. Such neuron- or neural stem cell-based therapy of diabetes is suggested following the spectacular results of Kuwabara et al [45] that raised the possibility of neural stem cells isolated from the adult brain functionally replacing beta cells in diabetic patients [83, 84]. The suggested workflow for autologous neural stem cell-based therapy for diabetes is, critically, based on the suggestion that insulinexpressing neural stem cells of the dentate gyrus or the olfactory bulb might find similar molecular niches for their survival and insulin-expressing ability in the brain as well as in the pancreas, and that this might involve Wnt3 and neurogenic differentiation 1 (NeuroD) [45, 84]. Neural stem cells can be isolated from rodent and human olfactory bulbs $[45,85]$ and rat cells can be transplanted directly into the pancreas of diabetic rats [45], where the pancreatic niche reprograms neuronal stem cells via Wnt signalling to express insulin. Isolating neural stem cells from models of type 1 diabetes (induced by streptozotocin in rats) or type 2 diabetes (in Goto-Kakizaki rats) followed by transplantation to the pancreas of animals of the corresponding model confirmed that grafted cells survive and produce insulin for long periods ( $>10$ weeks) and dramatically reduce blood glucose levels [45]. The therapeutic potential for human diabetic patients is immense because no genetic manipulation is necessary and the procedure bypasses tumorigenic pluripotent stem cells and concerns inherent to chronic immunosuppression.

\section{Summary and conclusion}

The action of insulin is not restricted to peripheral organs. Insulin receptors and signal transduction pathways described in the periphery are involved in a wide array of functions in the central nervous system. It is generally accepted that insulin produced by pancreatic beta cells in physiological conditions or applied intranasally with a therapeutic purpose for mild to moderate Alzheimer's disease finds its way to neurons of the cerebral cortex. The timescale of external insulin transport to the vicinity of neurons is relatively slow, consistent with longterm homeostatic regulation of neural networks. Recent work has overwhelmingly shown that insulin is also synthesised locally in the cerebral cortex. Neuron-derived insulin is capable of rapid modulation of synaptic and microcircuit mechanisms and is suggested to regulate on-demand energy homeostasis of neural networks. We suggest that novel therapeutic strategies might include modulation of neural insulin production in the brain by GLP-1 agonists for counteracting diabetes, obesity and neurodegenerative diseases. Recent experiments in which lost pancreatic beta cells were replaced by autologous transplants of insulin-producing neural progenitor cells signal the immense therapeutic potential of this approach for diabetes.

Funding Work in the authors' laboratory is supported by the European Research Council Interimpact project, the Hungarian Academy of Sciences and by the National Brain Research Program, Hungary.

Duality of interest The authors declare that there is no duality of interest associated with this manuscript.

Contribution statement Both authors were responsible for drafting the article and revising it critically for important intellectual content. Both authors approved the version to be published. 


\section{References}

1. Banting FG, Best $\mathrm{CH}$ (1922) The internal secretion of the pancreas. J Lab Clin Med 7:465-480

2. Mueckler M (1994) Facilitative glucose transporters. Eur J Biochem 219:713-725

3. Havrankova J, Roth J, Brownstein M (1978) Insulin receptors are widely distributed in the central nervous system of the rat. Nature 272:827-829

4. Wozniak M, Rydzewski B, Baker SP, Raizada MK (1993) The cellular and physiological actions of insulin in the central nervous system. Neurochem Int 22:1-10

5. Unger J, McNeill TH, Moxley RT 3rd et al (1989) Distribution of insulin receptor-like immunoreactivity in the rat forebrain. Neuroscience 31:143-157

6. Saltiel AR, Kahn CR (2001) Insulin signalling and the regulation of glucose and lipid metabolism. Nature 414:799-806

7. Niswender KD, Morrison CD, Clegg DJ et al (2003) Insulin activation of phosphatidylinositol 3-kinase in the hypothalamic arcuate nucleus: a key mediator of insulin-induced anorexia. Diabetes 52: 227-231

8. Banks WA, Owen JB, Erickson MA (2012) Insulin in the brain: there and back again. Pharmacol Ther 136:82-93

9. Gerozissis K (2010) The brain-insulin connection, metabolic diseases and related pathologies. In: Craft S (ed) Diabetes, insulin and Alzheimer's disease. Springer, Berlin, pp 21-42

10. Gray SM, Meijer RI, Barrett EJ (2014) Insulin regulates brain function, but how does it get there? Diabetes 63:3992-3997

11. McNay EC, Recknagel AK (2011) Brain insulin signaling: a key component of cognitive processes and a potential basis for cognitive impairment in type 2 diabetes. Neurobiol Learn Mem 96:432-442

12. Ghasemi R, Haeri A, Dargahi L et al (2013) Insulin in the brain: sources, localization and functions. Mol Neurobiol 47:145-171

13. Gerozissis K, Kyriaki G (2003) Brain insulin: regulation, mechanisms of action and functions. Cell Mol Neurobiol 23:1-25

14. Bondareva VM, Chistyakova OV (2007) Insulin and insulinreceptor signaling in the brain. Neurochem J 1:176-187

15. Margolis RU, Altszuler N (1967) Insulin in the cerebrospinal fluid. Nature 215:1375-1376

16. Banks WA, Jaspan JB, Kastin AJ (1997) Selective, physiological transport of insulin across the blood-brain barrier: novel demonstration by species-specific radioimmunoassays. Peptides 18:12571262

17. Strubbe JH, Porte D, Woods SC (1988) Insulin responses and glucose levels in plasma and cerebrospinal fluid during fasting and refeeding in the rat. Physiol Behav 44:205-208

18. Wallum BJ, Taborsky GJ, Porte D et al (1987) Cerebrospinal fluid insulin levels increase during intravenous insulin infusions in man. J Clin Endocrinol Metab 64:190-194

19. Kern W, Benedict C, Schultes B et al (2006) Low cerebrospinal fluid insulin levels in obese humans. Diabetologia 49:2790-2792

20. Schwartz MW, Sipols A, Kahn SE et al (1990) Kinetics and specificity of insulin uptake from plasma into cerebrospinal fluid. Am J Physiol 259:E378-383

21. Dietrich MO, Spuch C, Antequera D et al (2008) Megalin mediates the transport of leptin across the blood-CSF barrier. Neurobiol Aging 29:902-912

22. Orlando RA, Rader K, Authier F et al (1998) Megalin is an endocytic receptor for insulin. J Am Soc Nephrol 9:1759-1766

23. Genders AJ, Frison V, Abramson SR, Barrett EJ (2013) Endothelial cells actively concentrate insulin during its transendothelial transport. Microcirculation 20:434-439

24. Wang H, Wang AX, Barrett EJ (2011) Caveolin-1 is required for vascular endothelial insulin uptake. Am J Physiol Endocrinol Metab 300:E134-144
25. Wang H, Wang AX, Liu Z, Barrett EJ (2008) Insulin signaling stimulates insulin transport by bovine aortic endothelial cells. Diabetes 57:540-547

26. Wang H, Wang AX, Aylor K, Barrett EJ (2013) Nitric oxide directly promotes vascular endothelial insulin transport. Diabetes 62:4030 4042

27. Iliff JJ, Wang M, Liao Y et al (2012) A paravascular pathway facilitates CSF flow through the brain parenchyma and the clearance of interstitial solutes, including amyloid $\beta$. Sci Transl Med 4:147ra111

28. Orosco M, Gerozissis K, Rouch C, Nicolaïdis S (1995) Feedingrelated immunoreactive insulin changes in the PVN-VMH revealed by microdialysis. Brain Res 671:149-158

29. Gerozissis K, Orosco M, Rouch C, Nicolaidis S (1997) Insulin responses to a fat meal in hypothalamic microdialysates and plasma. Physiol Behav 62:767-772

30. Born J, Lange T, Kern W et al (2002) Sniffing neuropeptides: a transnasal approach to the human brain. Nat Neurosci 5:514-516

31. Banks WA, Kastin AJ (1998) Differential permeability of the blood-brain barrier to two pancreatic peptides: insulin and amylin. Peptides 19:883-889

32. Pardridge WM, Eisenberg J, Yang J (1985) Human blood-brain barrier insulin receptor. J Neurochem 44:1771-1778

33. Reger MA, Watson GS, Green PS et al (2008) Intranasal insulin improves cognition and modulates beta-amyloid in early AD. Neurology 70:440-448

34. Craft S, Baker LD, Montine TJ et al (2012) Intranasal insulin therapy for Alzheimer disease and amnestic mild cognitive impairment: a pilot clinical trial. Arch Neurol 69:29-38

35. Steen E, Terry BM, Rivera EJ et al (2005) Impaired insulin and insulin-like growth factor expression and signaling mechanisms in Alzheimer's disease - is this type 3 diabetes? J Alzheimers Dis 7: 63-80

36. Craft S, Peskind E, Schwartz MW et al (1998) Cerebrospinal fluid and plasma insulin levels in Alzheimer's disease: relationship to severity of dementia and apolipoprotein E genotype. Neurology 50:164-168

37. Lochhead JJ, Thorne RG (2012) Intranasal delivery of biologics to the central nervous system. Adv Drug Deliv Rev 64:614-628

38. Salameh TS, Bullock KM, Hujoel IA et al (2015) Central nervous system delivery of intranasal insulin: mechanisms of uptake and effects on cognition. J Alzheimers Dis 47:715-728

39. Havrankova J, Schmechel D, Roth J, Brownstein M (1978) Identification of insulin in rat brain. Proc Natl Acad Sci U S A 75:5737-5741

40. Baskin DG, Stein LJ, Ikeda H et al (1985) Genetically obese Zucker rats have abnormally low brain insulin content. Life Sci 36:627633

41. Banks WA (2004) The source of cerebral insulin. Eur J Pharmacol 490:5-1242

42. Di Guglielmo GM, Drake PG, Baass PC et al (1998) Insulin receptor internalization and signalling. Mol Cell Biochem 182:59-63

43. Dorn A, Bernstein HG, Rinne A et al (1983) Insulin- and glucagonlike peptides in the brain. Anat Rec 207:69-77

44. Dorn A, Rinne A, Bernstein HG et al (1983) Insulin and C-peptide in human brain neurons (insulin/C-peptide/brain peptides/immunohistochemistry/radioimmunoassay). J Hirnforsch 24:495-499

45. Kuwabara T, Kagalwala MN, Onuma Y et al (2011) Insulin biosynthesis in neuronal progenitors derived from adult hippocampus and the olfactory bulb. EMBO Mol Med 3:742-754

46. Birch NP, Christie DL, Renwick AG (1984) Proinsulin-like material in mouse foetal brain cell cultures. FEBS Lett 168:299-302

47. Devaskar SU, Singh BS, Carnaghi LR et al (1993) Insulin II gene expression in rat central nervous system. Regul Pept 48:55-63

48. Devaskar SU, Giddings SJ, Rajakumar PA et al (1994) Insulin gene expression and insulin synthesis in mammalian neuronal cells. J Biol Chem 269:8445-8454 
49. Deltour L, Leduque P, Blume N et al (1993) Differential expression of the two nonallelic proinsulin genes in the developing mouse embryo. Proc Natl Acad Sci U S A 90:527-531

50. Mehran AE, Templeman NM, Brigidi GS et al (2012) Hyperinsulinemia drives diet-induced obesity independently of brain insulin production. Cell Metab 16:723-737

51. Faragó N, Kocsis ÁK, Lovas S et al (2013) Digital PCR to determine the number of transcripts from single neurons after patchclamp recording. Biotechniques 54:327-336

52. Molnár G, Faragó N, Kocsis ÁK et al (2014) GABAergic neurogliaform cells represent local sources of insulin in the cerebral cortex. J Neurosci 34:1133-1137

53. Vannucci SJ, Koehler-Stec EM, Li K et al (1998) GLUT4 glucose transporter expression in rodent brain: effect of diabetes. Brain Res 797:1-11

54. Reagan LP (2005) Neuronal insulin signal transduction mechanisms in diabetes phenotypes. Neurobiol Aging 26(Suppl 1):56-59

55. Hirvonen J, Virtanen KA, Nummenmaa L et al (2011) Effects of insulin on brain glucose metabolism in impaired glucose tolerance. Diabetes 60:443-447

56. Baker LD, Cross DJ, Minoshima $\mathrm{S}$ et al (2011) Insulin resistance and Alzheimer-like reductions in regional cerebral glucose metabolism for cognitively normal adults with prediabetes or early type 2 diabetes. Arch Neurol 68:51-57

57. Bingham EM, Hopkins D, Smith D et al (2002) The role of insulin in human brain glucose metabolism: an 18fluoro-deoxyglucose positron emission tomography study. Diabetes 51:3384-3390

58. Emmanuel Y, Cochlin LE, Tyler DJ et al (2013) Human hippocampal energy metabolism is impaired during cognitive activity in a lipid infusion model of insulin resistance. Brain Behav 3:134144

59. McNay EC, Ong CT, McCrimmon RJ et al (2010) Hippocampal memory processes are modulated by insulin and high-fat-induced insulin resistance. Neurobiol Learn Mem 93:546-553

60. Gregg EW, Yaffe K, Cauley JA et al (2000) Is diabetes associated with cognitive impairment and cognitive decline among older women? Study of Osteoporotic Fractures Research Group. Arch Intern Med 160:174-180

61. Elias PK, Elias MF, D'Agostino RB et al (1997) NIDDM and blood pressure as risk factors for poor cognitive performance. The Framingham Study. Diabetes Care 20:1388-1395

62. Craft S, Watson GS (2004) Insulin and neurodegenerative disease: shared and specific mechanisms. Lancet Neurol 3: 169-178

63. Chiu S-L, Cline HT (2010) Insulin receptor signaling in the development of neuronal structure and function. Neural Dev 5:7

64. Beattie EC, Carroll RC, Yu X et al (2000) Regulation of AMPA receptor endocytosis by a signaling mechanism shared with LTD. Nat Neurosci 3:1291-1300

65. Lin JW, Ju W, Foster K et al (2000) Distinct molecular mechanisms and divergent endocytotic pathways of AMPA receptor internalization. Nat Neurosci 3:1282-1290

66. van der Heide LP, Kamal A, Artola A et al (2005) Insulin modulates hippocampal activity-dependent synaptic plasticity in a N-methyld-aspartate receptor and phosphatidyl-inositol-3-kinase-dependent manner. J Neurochem 94:1158-1166
67. Wan Q, Xiong ZG, Man HY et al (1997) Recruitment of functional GABA(A) receptors to postsynaptic domains by insulin. Nature 388:686-690

68. Jin Z, Jin Y, Kumar-Mendu S et al (2011) Insulin reduces neuronal excitability by turning on $\mathrm{GABA}(\mathrm{A})$ channels that generate tonic current. PLoS One 6:e16188

69. Tamas G, Lorincz A, Simon A, Szabadics J (2003) Identified sources and targets of slow inhibition in the neocortex. Science 299:1902-1905

70. Olah S, Fule M, Komlosi G et al (2009) Regulation of cortical microcircuits by unitary GABA-mediated volume transmission. Nature 461:1278-1281

71. Spanswick D, Smith MA, Mirshamsi S et al (2000) Insulin activates ATP-sensitive $\mathrm{K}+$ channels in hypothalamic neurons of lean, but not obese rats. Nat Neurosci 3:757-758

72. Stefani MR, Gold PE (2001) Intrahippocampal infusions of K-ATP channel modulators influence spontaneous alternation performance: relationships to acetylcholine release in the hippocampus. J Neurosci 21:609-614

73. Piroli GG, Grillo CA, Reznikov LR et al (2007) Corticosterone impairs insulin-stimulated translocation of GLUT4 in the rat hippocampus. Neuroendocrinology 85:71-80

74. Hamilton A, Hölscher C (2009) Receptors for the incretin glucagon-like peptide-1 are expressed on neurons in the central nervous system. Neuroreport 20:1161-1166

75. Korol SV, Jin Z, Babateen O, Birnir B (2015) GLP-1 and exendin-4 transiently enhance GABAA receptor-mediated synaptic and tonic currents in rat hippocampal CA3 pyramidal neurons. Diabetes 64: 79-89

76. Holst JJ (2007) The physiology of glucagon-like peptide 1. Physiol Rev 87:1409-1439

77. Lovshin JA, Drucker DJ (2009) Incretin-based therapies for type 2 diabetes mellitus. Nat Rev Endocrinol 5:262-269

78. Anthony K, Reed LJ, Dunn JT et al (2006) The cerebral basis for impaired control of food intake in metabolic syndrome. Diabetes 55:2986-2992

79. Heni M, Kullmann S, Preissl H et al (2015) Impaired insulin action in the human brain: causes and metabolic consequences. Nat Rev Endocrinol 11:701-711

80. Kleinridders A, Ferris HA, Cai W, Kahn CR (2014) Insulin action in brain regulates systemic metabolism and brain function. Diabetes 63:2232-2243

81. McClean PL, Hölscher C (2014) Liraglutide can reverse memory impairment, synaptic loss and reduce plaque load in aged APP/PS1 mice, a model of Alzheimer's disease. Neuropharmacology 76:57-67

82. Duarte AI, Candeias E, Correia SC et al (2013) Crosstalk between diabetes and brain: glucagon-like peptide- 1 mimetics as a promising therapy against neurodegeneration. Biochim Biophys Acta 1832:527-541

83. Basak O, Clevers H (2011) Neural stem cells for diabetes cell-based therapy. EMBO Mol Med 3:698-700

84. Kuwabara T, Asashima M (2012) Regenerative medicine using adult neural stem cells: the potential for diabetes therapy and other pharmaceutical applications. J Mol Cell Biol 4:1-2

85. Pagano SF, Impagnatiello F, Girelli M et al (2000) Isolation and characterization of neural stem cells from the adult human olfactory bulb. Stem Cells 18:295-300 